\title{
NEURAL FIBROSIS AND THE EFFECT OF NEUROLYSIS
}

\author{
MINORU SAKURAI, YOSHINORI MIYASAKA
}

From Tohoku University School of Medicine, Sendai, Japan

\begin{abstract}
Thickening of the fibrous element of a peripheral nerve may be caused by repeated friction, traction, constriction, ischaemia or partial rupture. The sequel may be a conduction disorder and a clinical condition such as an entrapment neuropathy or a tardy nerve palsy. Neural fibrosis is typically associated with a pseudoneuroma in continuity which has resulted from scarring and adhesions around the nerve as well as proliferation of the fibrous element within the nerve; the fibrosis may be classified as extraneural, intraneural or dispersive.

We report 17 cases treated by external neurolysis, with 14 satisfactory results, and 42 patients treated by internal neurolysis with success in 37 . Seven of the eight failures were in cases of dispersive fibrosis. A technique of internal neurolysis is described.
\end{abstract}

Neurolysis aims to remove or release fibrotic or scar tissue which is constricting a peripheral nerve. External neurolysis. a simple dissection to free the nerve from the encasing scar, is reported to be very effective (Seddon 1954: Bateman 1962: Sunderland 1968). Internal neurolysis or endoneurolysis - in which the nerve sheath is split. perifascicular scar tissue is dissected and individual fascicles within the nerve trunk are freed-is less reliably effective (Seddon 1972: Sunderland 1978). However, H. A. Brown (1970) and B. A. Brown (1972) both reported success in cases of post-traumatic fibrosis, as did Enna and Jacobson (1974) in leprous fibrosis. This paper describes the effect of neurolysis in 59 cases, with particular reference to simple fibrosis of the ulnar nerve at the elbow. including delayed ulnar nerve palsy. The mechanism of recovery of function is discussed.

Types of neural fibrosis. Fibrosis with adhesion to the tissues surrounding a nerve trunk is termed extra-neural fibrosis (Fig. 1). Fibrotic thickening and collagenous proliferation occurring in the epineurium or within the nerve trunk is intraneural fibrosis, and may be classified into epineurial, perineurial and endoneurial types. When a nerve trunk is crushed or partially ruptured, some axons are interrupted, and a neural scar is then formed at the site of the damage; this scar will include regenerating axons intermingled in a random pattern, and this is termed dispersive fibrosis.

M. Sakurai, MD. Associate Professor

Y. Miyasaka, MD. Instructor

Department of Orthopaedic Surgery. Tohoku University School of Medicine. I-I Seiryomachi. Sendai, 980 Japan.

Requests for reprints should be sent to Professor M. Sakurai

1. 1986 British Editorial Society of Bone and Joint Surgery

$0301620 \times 863068 \$ 2.00$

\section{MATERIAL AND METHODS}

We report 59 cases in which neurolysis was performed (Table I), externally in 17 and internally in 42. All the patients had some clinical features of neural involve-
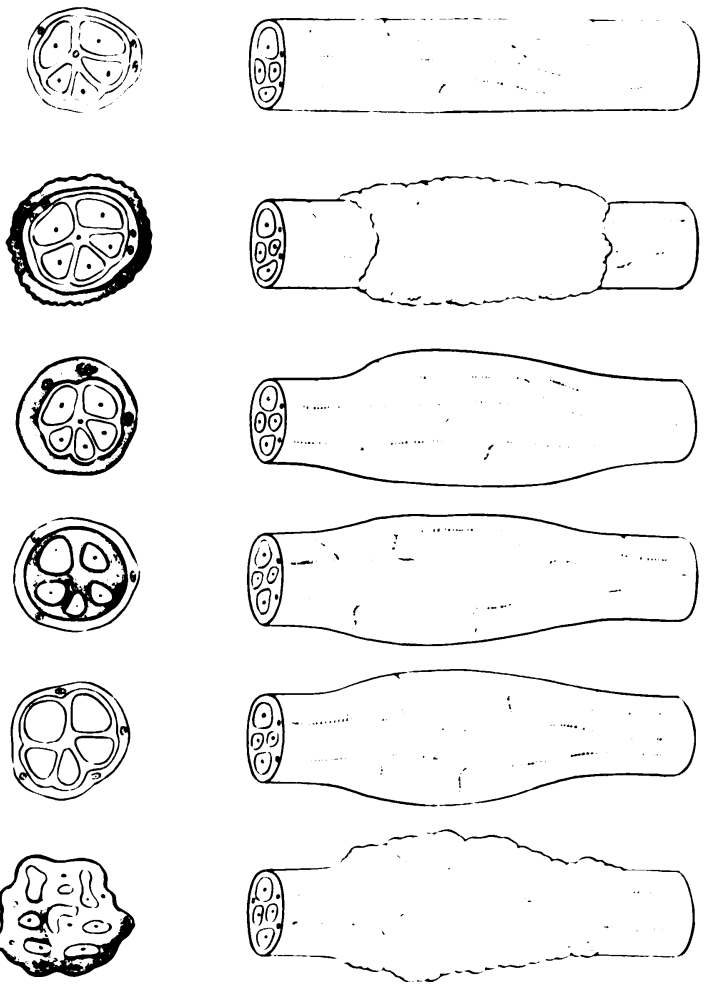

Fig. 1

Classification of neural fibrosis. The shaded areas indicate fibrosis. From ahove downwards:

Normal nerve

Fibrosis- Extraneural type

Intraneural, epineurial type Intraneural, perineurial type

Intraneural. endoneurial type

Dispersive type. 


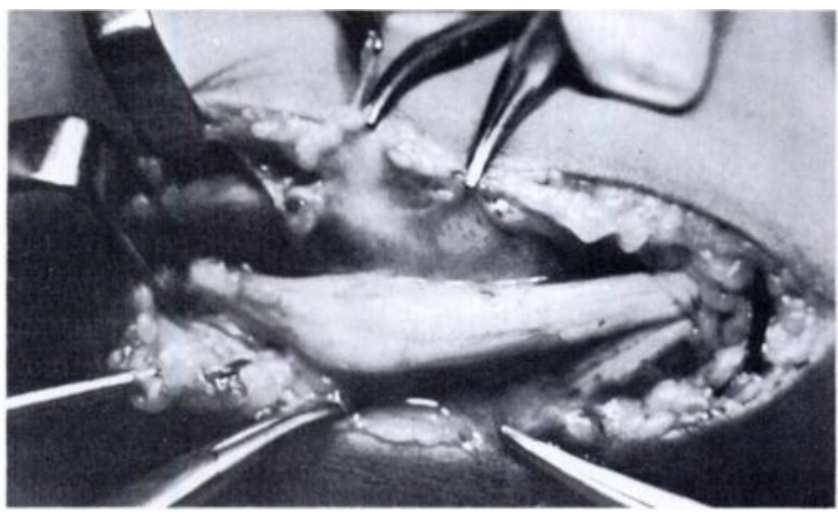

Fig. 2

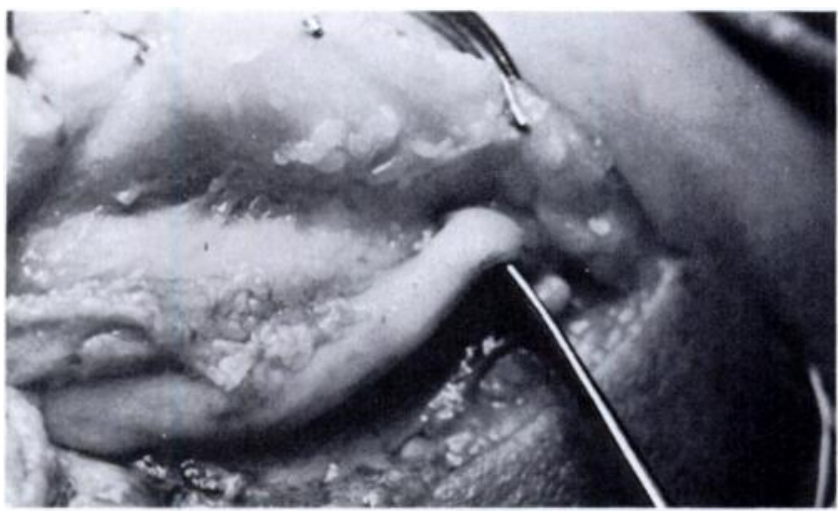

Fig. 3

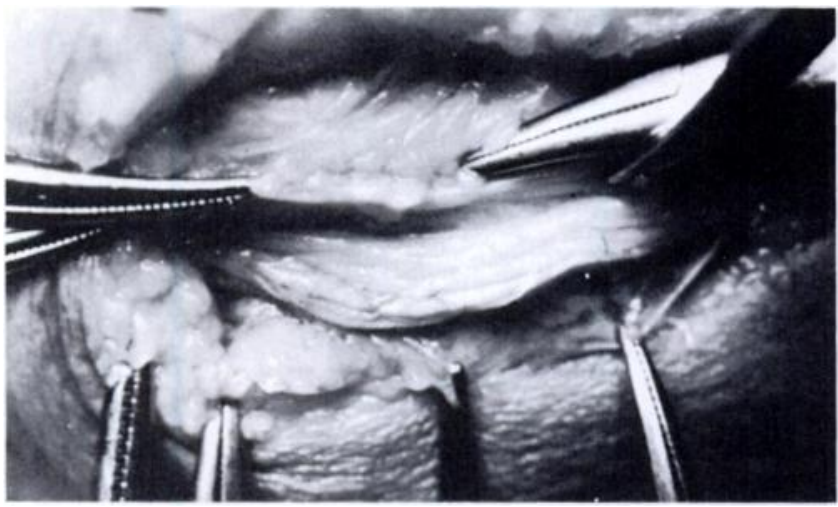

Fig. 5
Figure 2 A typical pseudoneuroma of the ulnar nerve due to friction neuritis. It is proximal to Osborne's band, which has been divided, on the left of the photograph. Figures 3 and 4 To show the differing amounts of flexibility of the trunk of the ulnar nerve when it is lifted proximal to the fibrosis (Fig. 3), and in the region of neural fibrosis (Fig. 4) where it is seen and felt to be stiff. Figure 5- Tough epineurium has been incised longitudinally and retracted laterally. Figure 6 - When the tight perineurium is incised. the compressed neural element extrudes.

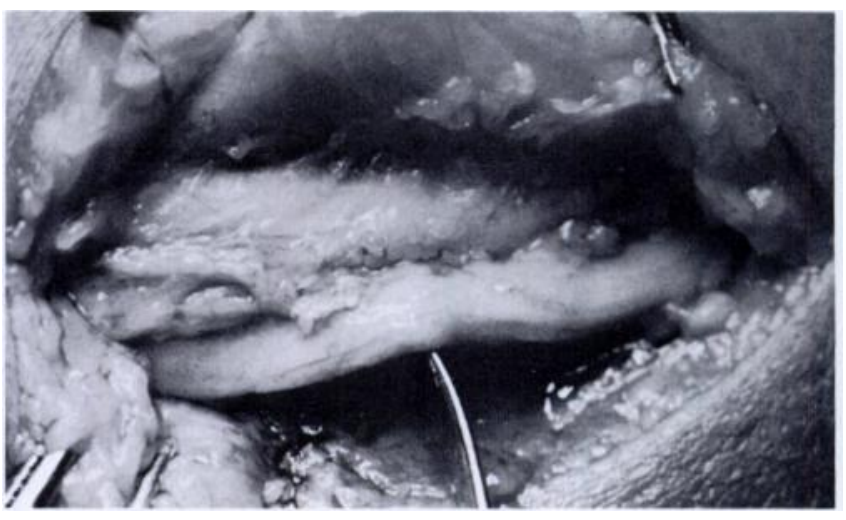

Fig. 4

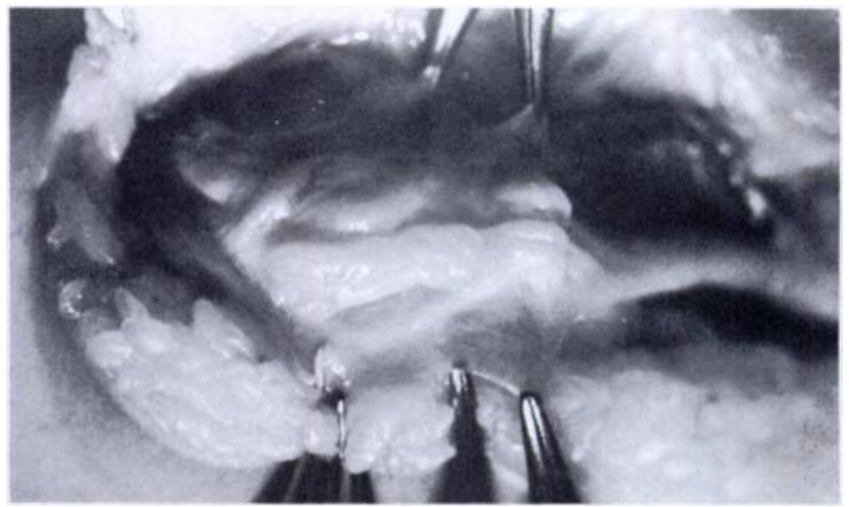

Fig. 6 
Table $\mathbf{l}$. The nerve involved and the type of neurolysis performed in 59 cases. The cases in which dispersive fibrosis was found are shown in parentheses

\begin{tabular}{lccc} 
& \multicolumn{2}{c}{ Neurolysis } & \\
\cline { 2 - 3 } Nerve & External & Internal & Total \\
\hline Brachial plexus & $6(2)$ & 2 & 8 \\
Radial & & 1 & 1 \\
Median & 5 & $2(1)$ & 7 \\
Ulnar & 2 & $27(2)$ & 29 \\
Sciatic & - & $4(1)$ & 4 \\
Femoral & 1 & & 1 \\
Lateral femoral cutaneous & 1 & & 1 \\
Common peroneal & 1 & 4 & 5 \\
Tibial & & $2(1)$ & 2 \\
Sural & 1 & - & 1 \\
Total & 17 & 42 & 59
\end{tabular}

ment, but continuity of the nerve had been demonstrated by methods such as electromyography or sweat testing.

In 10 patients the brachial plexus was explored; external neurolysis was done in eight, two of which had dispersive fibrosis due to severe crush injury, and internal neurolysis in two. Nearly half of our neurolyses were of the ulnar nerve, 27 of the 29 being for simple fibrotic change; in most of these there was either a friction neuritis with constriction by a fibrous band (Osborne 1957), or a very chronic stretch lesion produced by extreme cubitus valgus secondary to supracondylar fracture in childhood. Neurolyses were also performed on the radial, median, sciatic, femoral, lateral femoral cutaneous, common peroneal, tibial and sural nerves.

Operation. A typical pseudoneuroma is shown in Figure 2 , with enlargement of the ulnar nerve proximal to the site of constriction and reduced elasticity of its trunk. Operation on the ulnar nerve at the elbow was most often carried out under local anaesthesia, using $0.5 \%$ Xylocaine with adrenaline. Blood vessels along the nerve trunk were carefully preserved when they were dissected from surrounding fatty or connective tissue. Inspection and palpation are important to identify the site of any rigidity in the nerve trunk and this is helped by observation of flexibility while lifting the nerve with a blunt instrument (Figs 3 and 4). These features and the stiff texture of the pseudoneuroma indicate proliferation of the fibrous element of the nerve trunk. In most cases a small amount of saline was injected with a fine needle into and around the epineurium before this was incised. The tip of a scalpel was then used for repeated fine cuts, carefully and gently, along the distended fibrous tissue in a longitudinal direction until the perineurium was exposed. Extrinsic and intrinsic vessels are preserved as far as possible. Local anaesthetic need not be injected into the neural element, since pain is not provoked until the tip of the scalpel encounters nerve fibres. When the epineurium is thick and tough, it can very easily be peeled aside, retracted laterally from the line of the incision, and held with fine haemostats (Fig. 5). If there had been severe constriction by epineural fibrosis, the contents of the nerve tend to bulge. Their texture is carefully observed and palpated with a fingertip; if the perineurium is fibrotic and tight, then it is cut by a similar precise manoeuvre.

The neural element is a glossy, dark-yellow colour and protrudes from the cleft of the incised perineurium (Fig. 6). Bulging at this level indicates that the neural element has been under considerable pressure.

The technique of internal neurolysis is shown in Figure 7. If the scalpel touches sensory nerve fibres within a fascicle it may cause peripheral tingling pain, while stimulation of a motor nerve fibre may evoke a muscle twitch. These alarm responses, preserved by operating under local anaesthesia, help to minimise damage to nerve axons.

Similar precise incisions may be needed throughout the zone of neural fibrosis as well as into the pseudoneuroma, but the sacrifice of any extrinsic and intrinsic small vessels should be avoided. When internal neurolysis has been completed, the fibrotic zone of the nerve will feel softer and much more elastic.

When there is a pseudoneuroma proximal to the fibrotic fascia of the flexor carpi ulnaris muscle (Osborne's band), this constriction is first incised to open the narrowed cubital tunnel. For friction neuritis of the ulnar nerve or delayed ulnar nerve palsy, we prefer epicondylotomy (King and Morgan 1959), reflecting the periosteum to expose the bony tissue of the epicondyle and trimming the bone with an osteotome, then suturing the periosteum. This technique flattens the epicondyle and allows the ulnar nerve to shift medially and anter-

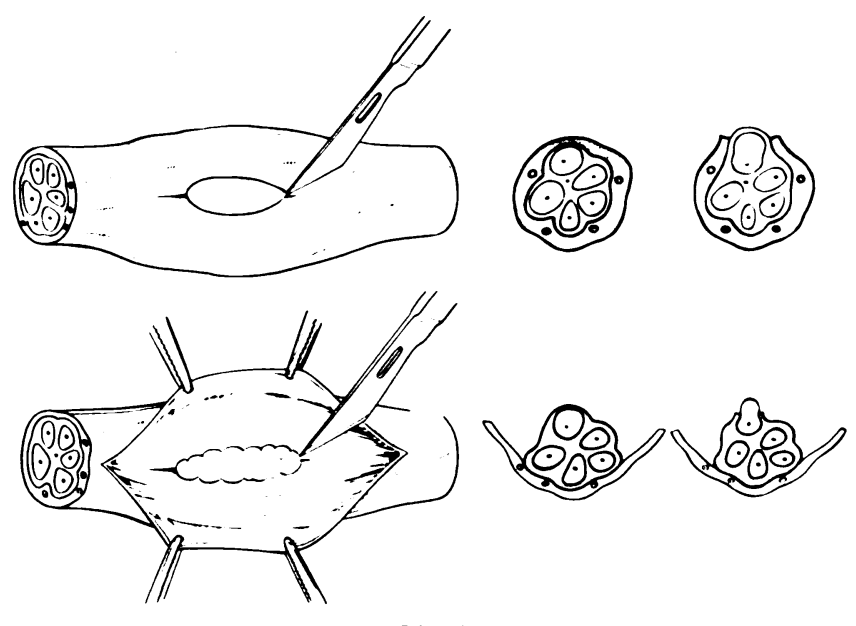

Fig. 7

Drawing to show the technique of internal neurolysis. 
iorly, eliminating friction and tightness of the nerve during movement of the elbow.

Internal neurolysis of other nerves, such as for pseudoneuroma of the median nerve in carpal tunnel syndrome after release of the transverse ligament, was carried out in the same manner, though sometimes different types of anaesthesia were used. Internal neurolysis was not, however, applicable to the brachial plexus because the fascicular pattern in the plexus is so complex that incision or dissection of perineurium would probably damage neural axons even in a short segment of cord, branch or trunk. In most cases of brachial plexus lesion with dense fibrosis, only epineurotomy was performed.

After crush injury or partial severance of a nerve trunk, dense neural scar is formed. This makes it difficult to identify epineurium and perineurium, and therefore to carry out an internal neurolysis. In general, external neurolysis only was used to release the fibrotic scar after such a nerve had been dissected from surrounding adhesions.

Clinical evaluation. Motor disability and sensory disturbance were carefully assessed clinically before operation. In patients with ulnar nerve palsy, the presence of claw hand, Froment's sign, the crossed-finger test (Earle and Vlastou 1980) and two-point discrimination at the finger pulp were investigated. Sweating function was tested by the bromphenol blue printing method (Sakurai 1974), but in almost all the ulnar nerve palsy patients there was hardly any diminution.

Maximal conduction velocity was measured electrically in the usual manner. Motor nerve conduction velocity was measured precisely, especially in the region of the elbow, moving the stimulating electrode only a few centimetres at a time. Evoked potentials were recorded from the abductor digiti minimi or other intrinsic muscles innervated by the ulnar nerve.

\section{RESULTS}

These are listed in Table II. Internal neurolysis was carried out for simple neural fibrosis in 37 cases, whether or not there was a pseudoneuroma caused by friction or compression, and the results were undoubtedly satisfactory. Motor dysfunction and sensory disturbance varied from case to case, but improved muscle power, and especially a stronger grip, were found in all cases of ulnar nerve palsy when examined from one week to one month or more after operation. Recovery from sensory disturbance was obtained in most cases, but this was somewhat delayed. External neurolysis was effective in 14 of 15 cases. In seven cases of dispersive neural fibrosis, two external and five internal neurolyses were performed, but none obtained good recovery of nerve function.

In one patient with ulnar nerve palsy, there was an increase in power of grip from 10 to $30 \mathrm{~kg}$ on the day after operation; another recovered from claw-hand deformity one week after surgery.
The cases of dispersive fibrosis in which no improvement was obtained are listed in Table III. The causes of fibrosis included infiltration of the brachial plexus by desmoid fibroma, partial rupture by gunshot wound involving the median, ulnar and sciatic nerves and, in one case, a crush injury of the tibial nerve. Despite careful, and somewhat tedious and lengthy operations, no definite improvement followed neurolysis.

Table II. The numbers of cases with each type of neural fibrosis and the results of neurolysis

\begin{tabular}{llllll}
\hline & & \multicolumn{2}{l}{ Neurolysis } & & \multicolumn{2}{l}{\begin{tabular}{l} 
Result \\
\cline { 3 - 5 } Type
\end{tabular}} & Number & External & Internal & $\begin{array}{l}\text { Improve- } \\
\text { ment }\end{array}$ & $\begin{array}{l}\text { No } \\
\text { change }\end{array}$ \\
\hline Extraneural & 15 & 15 & & 14 & 1 \\
Intraneural & 37 & & 37 & 37 & \\
Dispersive & 7 & 2 & 5 & & 7 \\
$\quad$ Total & 59 & 17 & 42 & 51 & 8 \\
\hline
\end{tabular}

Table III. The nerve, site and cause of the seven cases of dispersive neural fibrosis

\begin{tabular}{lll}
\hline Nerve & Site & Cause \\
\hline Brachial plexus & & Fibroma infiltration \\
Brachial plexus & & Desmoid tumour \\
Median & Upper arm & Gunshot wound \\
Ulnar & Upper arm & Gunshot wound \\
Ulnar & Elbow & Partial division \\
Sciatic & Middle thigh & Gunshot wound \\
Tibial & Knee & Cut and crush injury \\
\hline
\end{tabular}

Cases of ulnar nerve palsy with slight neural disturbance showed delayed motor nerve conduction for only a few centimetres around the elbow joint before operation (Fig. 8), and an electromyogram showed spread or dispersion of the evoked potential especially from stimulation proximal to the involved zone (Fig. 9). Postoperative electrical examination showed a tendency to restoration of conduction velocity and to recovery of the pattern of evoked potential (Fig. 10).

Thus, not only external but also internal neurolysis has been shown to be useful for the simple neural fibrosis which frequently occurs in the ulnar nerve at the elbow as a result of mechanical friction, traction or local compression. Improvement is produced by the release of compression of the neural axons by freeing them from constricting fibrosis both outside and inside the nerve.

\section{DISCUSSION}

The classification of peripheral nerve injuries into neurotmesis, axonotmesis and neurapraxia (Seddon 1943), is generally accepted, but neurapraxia has many 
different causes, and a conduction block produced by compression may vary considerably with the magnitude and duration of the deforming force. Category 1, as classified by Sunderland (1968), is a temporary conduction block with immediate recovery after removal of the pressure; it is almost equivalent to Seddon's neurapraxia. A long-existing conduction block which recovers after decompression of the nerve fibres fits Sunderland's Categories 2 and 3.

On the other hand, there is the concept of axonostenosis proposed by Bauwens (1960) and based on electrical studies. He assumed that dysfunction of the neural axon was limited to the actual site of compression of axons in continuity. Similar evidence was obtained from our electrodiagnostic studies on ulnar nerve palsy, in
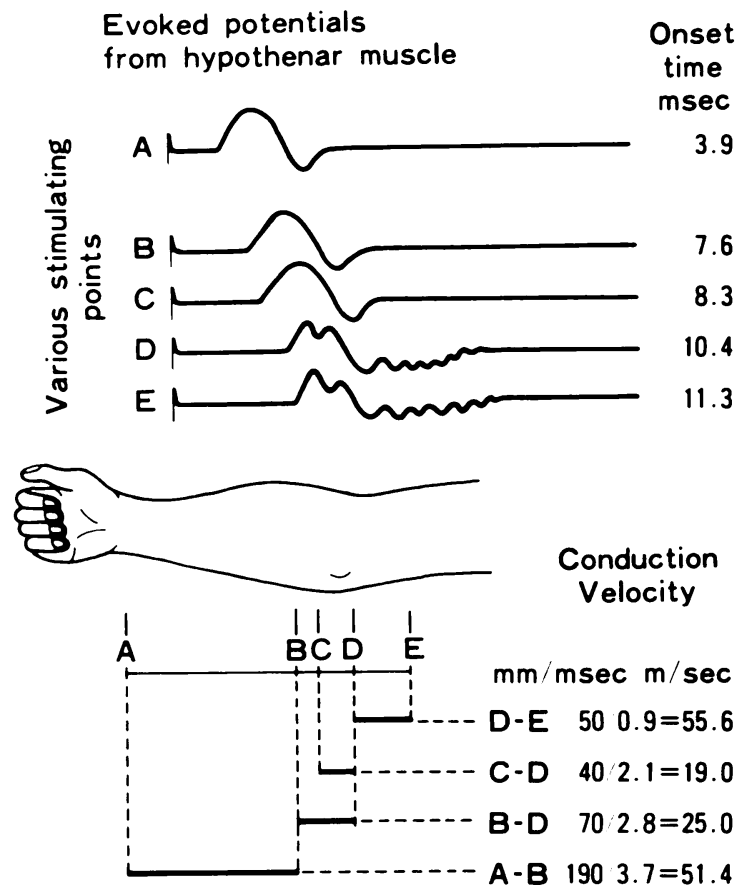

Fig. 8

Series of electromyograms of evoked potentials produced by changing the point of stimulation. The conduction velocity over the involved cubital portion of the nerve, between $B$ and $D$. is markedly slower. which there was a delay of conduction velocity only at the zone of compression caused by fibrosis.

Our results after internal neurolysis for neural fibrosis support the findings of H. A. Brown (1970) and B. A. Brown (1972). The surgical techniques reported by Curtis and Eversmann (1973), Enna and Jacobson (1974), and Spinner and Spencer (1974) are more extensive, involving exploration and dissection of each fascicle in the fibrotic region of the nerve trunk rather than simple epineurotomy or perineurotomy. Precise comparison of the results from different investigators is not easy, but we appear to have a higher rate of recovery than that reported by Curtis and Eversmann (1973).

The anatomy of intraneural microvessels in experimental animals was extensively studied by Lundborg (1975), and there is no doubt that the preservation of the blood supply to the nerve during operation is of importance. We took care to preserve the circulation during neurolysis. Even more meticulous handling, using a microscope, would be ideal, and might give better results.

Sunderland (1946) found that experimentally produced rupture of perineurium caused partial degeneration of the nerve fibres within a fascicle; he assumed that herniation of nerve fibres through a restricted rupture was likely to be the cause. A restricted type of rupture would be rare in a traumatic injury. At operation, once the neural element is seen to protrude from a small incision in the perineurium, the incision should be lengthened; if this is not done, kinking of the nerve fibres occurs. In our experience, compressed neural elements protrude obviously when the dense neural sheath is incised, showing that pressure in the axoplasm has been reduced, and allowing axonal flow to return to a more normal physiological condition.

Several theories have been proposed for the mechanism which causes dysfunction of a peripheral nerve when compressed. Experimentally, paralysis induced by direct pressure is thought to be due to ischaemia (DennyBrown and Brenner 1944). Similarly, Sunderland (1976) considered neurapraxia to be caused mainly by venous obstruction at the epineurium. Blood circulation is apparently necessary to maintain the nutritional and metabolic condition of axoplasm, and decompression of
Series of electromyograms of evoked potentials from the abductor digiti minimi muscle. These were produced by increasing strengths of stimulus to the ulnar nerve proximal to the involved cubital portion in a 53-year-old man with delayed ulnar nerve palsy. Figure 9Before operation, dispersion of the potential is seen as a result of moderate neuropathy. Figure 10-Three months after internal neurolysis, the dispersion has decreased. and the pattern of simple biphasic oscillation has almost returned to normal.

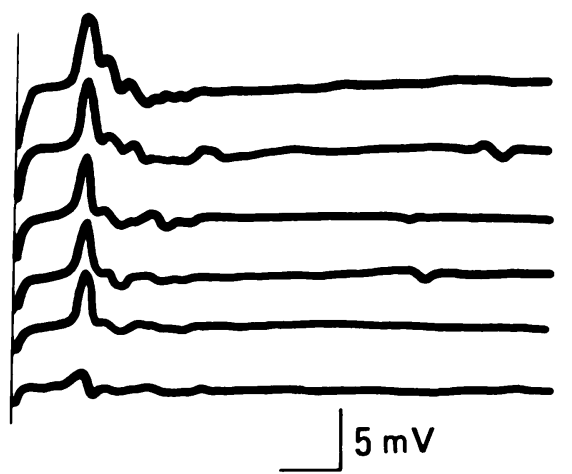

Fig. 9
$10 \mathrm{msec}$

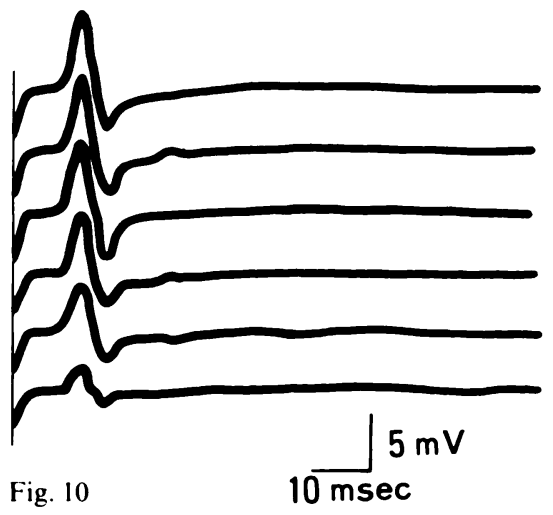


an involved nerve undoubtedly helps to restore its blood supply. On the other hand, Ochoa, Fowler and Gilliatt (1972) found that invagination of the myelin sheath at the nodes of Ranvier was produced by acute, severe transient compression from a pneumatic cuff. They also considered that bulbous and tapered myelin at internodal intervals could cause interruption of conductivity. These mechanisms might have been significant in some of the patients who showed immediate recovery of nerve function after neurolysis.

Operative decompression or neurolysis of a peripheral nerve aims to restore the conductivity of nerve fibres which have lost function because of deficiency of blood supply or disturbed axonal metabolism or both. Meticulous but accurate release of the pressure within a fibrotic section of a peripheral nerve may help not only to improve the blood supply and to restore vigorous axonal flow, but also to improve the field for regeneration of nerve fibres which have suffered axonotmesis.

\section{REFERENCES}

Bateman JE. Trauma to nerves in limbs. Philadelphia \& London: WB Saunders, 1962;167.70.

Bauwens P. Electrodiagnostic definition of the site and nature of peripheral nerve lesions. Ann Phys Med 1960:5:14952.

Brown BA. Internal neurolysis in traumatic peripheral nerve lesions in continuity. Surg Clin North Am 1972:52(5): 1167-75.

Brown HA. Internal neurolysis in the treatment of peripheral nerve injuries. Clin Neurosurg 1970:17:99-110.
Curtis RM, Eversmann WW Jr. Internal neurolysis as an adjunct to the treatment of the carpal-tunnel syndrome. J Bone Joint Surg $[\mathrm{Am}]$ 1973:55-A:733-40.

Denny-Brown D, Brenner C. Paralysis of nerve induced by direct pressure and by tourniquet. Arch Neurol Psychiat 1944;51:1-26.

Earle AS, Vlastou C. Crossed fingers and other tests of ulnar nerve motor function. $J$ Hand Surg 1980;5:560-5.

Enna CD, Jacobson RR. A clinical assessment of neurolysis for leprous involvement of the ulnar nerve. Int J Lepr 1974;42:162-4.

King T, Morgan FP. Late results of removing the medial humeral epicondyle for traumatic ulnar neuritis. J Bone Joint Surg [Br] 1959; 41- B:51- 5.

Lundborg G. Structure and function of the intraneural microvessels as related to trauma, edema formation, and nerve function. $J$ Bone Joint Surg $[\mathrm{Am}] 1975 ; 57-\mathrm{A}: 938-48$.

Ochoa J, Fowler TJ, Gilliatt RW. Anatomical changes in peripheral nerves compressed by a pneumatic tourniquet. J Anat 1972;113: 433-55.

Osborne GV. The surgical treatment of tardy ulnar neuritis. $J$ Bone Joint Surg [Br] 1957;39-B:782.

Sakurai M. Printing method for detecting the sweat employing bromphenol blue: its utility to determine the site of brachial plexus lesion (in Japanese). Jpn J Traum Occup Med (Nihon Saigai Igakkaishi) 1974;22:1066-74.

Seddon HJ. Three types of nerve injury. Brain 1943;66:237-88.

Seddon HJ, ed. Nerve Injuries Committee of the Medical Research Council. Peripheral Nerve Injuries. MRC Special Report Series 282. London: HMSO, 1954:81.

Seddon HJ. Surgical disorders of the peripheral nerves. Edinburgh and London: Churchill Livingstone, 1972:277-81.

Spinner M, Spencer PS. Nerve compression lesions of the upper extremity: a clinical and experimental review. Clin Orthop 1974; 104:46-67.

Sunderland S. The effect of rupture of the perineurium on the contained nerve-fibres. Brain 1946;69: 149-52.

Sunderland S. Nerves and nerve injuries. Ist ed. Baltimore: Williams \& Wilkins, 1968:558 62 .

Sunderland $\mathbf{S}$. The nerve lesion in the carpal tunnel syndrome. $J$ Neurol Neurosurg Psychiatry 1976;39:615-26.

Sunderland S. Nerves and nerve injuries. 2nd ed. Edinburgh etc: Churchill Livingstone, 1978;499-503. 